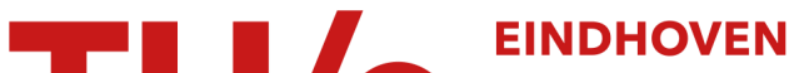 \\ UNIVERSITY OF \\ TECHNOLOGY
}

\section{Camera-based on-line short cessation of breathing detection}

\section{Citation for published version (APA):}

Lorato, I., Stuijk, S., Meftah, M., Verkruijsse, W., \& De Haan, G. (2019). Camera-based on-line short cessation of breathing detection. In Proceedings - 2019 International Conference on Computer Vision Workshop, ICCVW 2019 (pp. 1656-1663). [9022052] Institute of Electrical and Electronics Engineers.

https://doi.org/10.1109/ICCVW.2019.00205

DOI:

10.1109/ICCVW.2019.00205

Document status and date:

Published: 28/10/2019

\section{Document Version:}

Accepted manuscript including changes made at the peer-review stage

\section{Please check the document version of this publication:}

- A submitted manuscript is the version of the article upon submission and before peer-review. There can be important differences between the submitted version and the official published version of record. People interested in the research are advised to contact the author for the final version of the publication, or visit the $\mathrm{DOI}$ to the publisher's website.

- The final author version and the galley proof are versions of the publication after peer review.

- The final published version features the final layout of the paper including the volume, issue and page numbers.

Link to publication

\section{General rights}

Copyright and moral rights for the publications made accessible in the public portal are retained by the authors and/or other copyright owners and it is a condition of accessing publications that users recognise and abide by the legal requirements associated with these rights.

- Users may download and print one copy of any publication from the public portal for the purpose of private study or research.

- You may not further distribute the material or use it for any profit-making activity or commercial gain

- You may freely distribute the URL identifying the publication in the public portal.

If the publication is distributed under the terms of Article $25 f a$ of the Dutch Copyright Act, indicated by the "Taverne" license above, please follow below link for the End User Agreement:

www.tue.nl/taverne

Take down policy

If you believe that this document breaches copyright please contact us at:

openaccess@tue.nl

providing details and we will investigate your claim. 


\title{
Camera-based On-line Short Cessation of Breathing Detection
}

\author{
Ilde Lorato \\ Eindhoven University of Technology \\ Eindhoven, The Netherlands. \\ i.r.loratodtue.nl \\ Mohammed Meftah \\ Philips Research \\ Eindhoven, The Netherlands
}

\author{
Sander Stuijk
Eindhoven University of Technology \\ Sander Stuijk
Eindhoven University of Technology \\ Eindhoven, The Netherlands
}

Wim Verkruijsse

Philips Research

Eindhoven, The Netherlands

\author{
Gerard de Haan \\ Eindhoven University of Technology \\ Philips Research \\ Eindhoven, The Netherlands
}

\begin{abstract}
Apnea detection is extremely important in neonatal settings because hypoxia can lead to permanent impairment. Short cessations of breathing are very common in infants and could be used for example for the prediction of longer apneas. The aim of this study is to investigate the accuracy of our on-line cessation of breathing detector. Signals obtained through camera-based respiration monitoring were analyzed in five infants with 91 annotated cessations of breathing. The method proposed is based on the comparison of short-term and long-term standard deviations allowing the detection of sudden amplitude reduction in the signal with a low latency. A new strategy able to detect short cessations of breathing on-line was successfully validated yielding an average accuracy of $93 \%$.
\end{abstract}

\section{Introduction}

Vital signs are of critical value to check the health of premature infants. Their monitoring is therefore standard practice in Neonatal Intensive Care Units (NICUs). Since Apnea Of Prematurity (AOP) is common in this population, continuous respiration monitoring is crucial [11]. Apneas are prolonged pauses in the respiration and are common in infants with a gestational age below 34 weeks [12], the hypoxia typically associated with apnea could cause longterm or permanent impairment [14]. Respiration monitoring based on Chest Impedance (CI) currently used in NICUs presents limitations when detecting apneas, in particular, cardiac artifacts are a common cause of missed apnea de- tection [24], moreover, motion artifacts and other thoracic movements can also be misinterpreted as respiration [7]. Apneas are strictly defined as a Cessation Of Breathing (COB) longer than 20 seconds or a $\mathrm{COB}$ of 10 seconds accompanied by bradycardia and/or desaturation [28]. However, discussions on the definition of clinically relevant apneas move the focus also on shorter COB [13]. Short apneic episodes are common in infants and are defined as a respiratory pause of at least 3 seconds [8]. Moreover, this type of events can provide insights on the infant's respiratory system [33] and lead possibly to apnea prediction [6].

Since adhesive electrodes and sensors can cause stress or even skin damage to the infants' sensitive skin [1], research in this field has been focusing on alternative non-contact respiration monitoring techniques. Between these, radars [21, 15], RGB or Near InfraRed (NIR) cameras [35, 23, 22], vision system based on depth sensing [5, 29], thermal cameras $[27,2]$, and pressure sensitive mattress $[19,4]$ are the most researched for respiration monitoring in a NICU environment. Cameras represent one of the best solutions for NICUs applications. In the first place, because they are completely unobtrusive passive sensors and they allow to monitor multiple vital signs simultaneously. Moreover, cameras also provide contextual information that would be useful to nursing staff for infants' observation, and it would promote family-centred NICUs through live video feed to parents [31].

In this paper, we propose an approach for the on-line detection of short apneic events through camera-based respiration monitoring. Many methods for respiration detection algorithms using cameras have been proposed targeting 


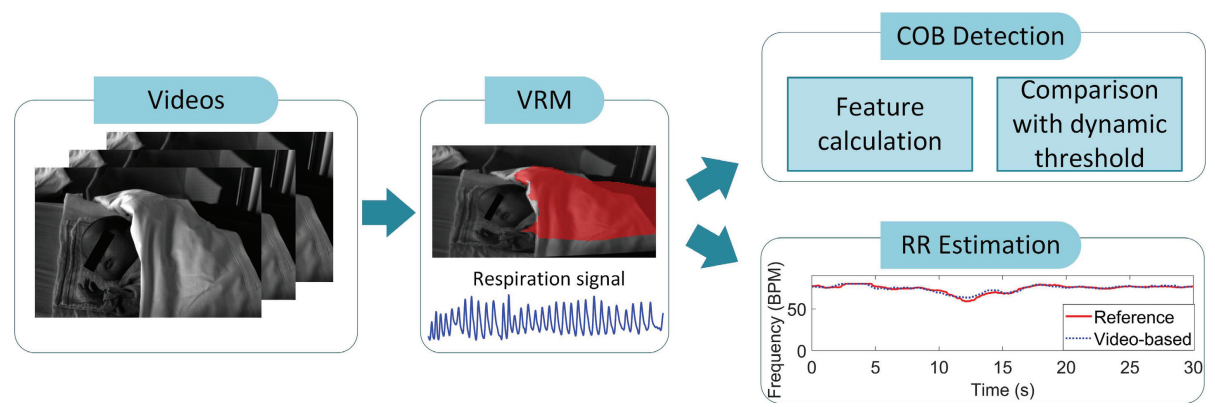

Figure 1. Main processing steps leading to the detection of Cessation Of Breathing (COB).

an infant population. Jorge et al. [18] proposed a camerabased approach for respiration monitoring based on a skin detection algorithm, which is not ideal for NICUs applications since infants' abdominal areas are commonly covered with blankets or snuggles. Though remote PPG-based approaches, e.g. [32], also rely on skin visibility, they can work on facial skin that is more likely uncovered. However, motion robustness necessitates multi-wavelength cameras. RGB-cameras are a seemingly logical choice, but visible illumination may disturb sleep and is therefore not allowed. Proposals using wavelengths in the infrared range suffer from high cost of multi-wavelength cameras, or parallax when using 3 cameras in parallel. Therefore, remote PPG-based solutions are not straightforward in such a complex environment. However, when monitoring respiration based on motion, skin visibility and color information are not indispensable for the signal detection. For example, Allinovi et al. [3] proposed a method based on maximum likelihood modeling and motion magnification able to automatically select the Region Of Interest (ROI). The method proposed was tested on a limited dataset of adults and infants videos, with a window size of 20 seconds for estimation of the respiration signal and the respiration rate. The latency (caused by the processing window) is particularly important when aiming at apnea detection and therefore, the method proposed by Janssen et al. [16] was preferred as a starting point for our work. The method, called Video Respiration Monitoring (VRM), was extensively tested on adults videos, but limited experiments were performed on infants. Still, we consider this method very appealing for NICU-applications, particularly because of its attractive automatic ROI detection independent on skin visibility and the low latency of the method.

The output of the VRM algorithm is used as starting point for the detection of short apneic events in the respiration signal. Other works have been focusing on apnea detection strategies starting from video extracted respiratory signals, Jorge et al. [17] proposed an approach based on camera where $\mathrm{COB}$ longer than 20 seconds were classified based on the Respiration Rates (RRs). If the RRs of the videos were lower than 20 breaths per minute for a period longer than $20 \mathrm{~s}$ and no other motion was present in the video segments then it was classified as an apnea. However, aiming at the detection of short apneas time-domain approaches are preferable being more sensitive to particularly short variations. Also Cattani et al. [9] tested camera-based respiration signal for the detection of apneas. The apnea detection strategy was to compare the time domain signal with an empirical constant threshold equal to 0.14 . Constant thresholds have the drawback of not being able to adapt dynamically to changes in the signals, such as reductions in amplitude. Lee et al. [24], instead, proposed an approach based on modeling the distribution of normal breathing patterns and apnea ones reaching an average detection performance over $90 \%$ by analysing chest impedance signals. The approach is, however, suitable only for retrospective analysis as specified by the authors, since the empirical parameters were optimized after filtering and baseline removal of the entire signal. This method has been widely used in apnea related publications, e.g. [26, 25, 10, 34], and has also been employed for the detection of short apneic events [6], therefore we decided to use it for comparison purposes.

The main contribution of this work is the development of an on-line short cessation of breathing detection strategy based on the comparison of the short-term standard deviation with the long-term standard deviation. The respiration rate is obtained as a byproduct of our processing. This is the first method able to detect short cessations of breathing with a low latency. The rest of the paper is organized as follows: Section 2 explains the method used and the dataset, Section 3 presents the results. Sections 4 and 5 contain respectively the discussion and the conclusion.

\section{Materials and methods}

\subsection{Method}

Figure 1 summarizes the principal steps of the processing algorithm. The NICU-videos are input to our processing. The VRM-algorithm of Janssen et al. (Section 2.1.1) is used to extract the respiratory signal. On this respiratory 
signal, we run our COB-detector described in Section 2.1.2. Additionally, we compute and output the respiratory rate. In our benchmarking, we shall compare our COB-detector with the results from Lee et al. [24], and the RR with the CI-reference. The proposed algorithm was implemented in MATLAB (MATLAB 2018b, The MathWorks Inc., Natick, MA, USA).

\subsubsection{Video respiration monitoring algorithm}

The VRM algorithm proposed by Janssen et al. in [16] is a respiration-motion detection algorithm based on Optical Flow (OF). The algorithm automatically detects the ROI for respiration detection and returns the respiration signal. When motion not related to the respiration is detected, the respiration waveform is put to zero and a template indicating that motion unrelated to respiration is present can be obtained. The same parameters introduced in the paper for the neonatal case were used in this work.

The CI signal and the respiration signal obtained from the videos are both filtered using a band-pass Butterworth filter of the $4^{t h}$ order between 30 and 80 Breaths Per Minutes (BPM) since this is the normal range of respiration rate in NICU infants including also tachypnea cases [30]. The signals have different sampling frequencies corresponding to 15 frames per second or 20 frames per second depending on the acquisition and are processed with a sliding window approach with a window size of 3 seconds and a slide of 1 frame.

\subsubsection{Cessation of breathing detection}

In case of a central apnea breathing cessation, a strong decrease in amplitude of the respiratory signal could be expected. Hence, our proposed COB-detector aims at signaling relative decreases in standard deviation of the respiratory signal. Such a decrease can be recognized, by the shortterm standard deviation $\sigma_{s}$ becoming significantly smaller than the long-term standard deviation $\sigma_{l}$. Parameters in such an approach are the window-lengths for computation of the two standard deviations, and the threshold to define if a drop is "significant". Therefore, two window lengths are defined: a short window $l_{s}$ in which a feature, corresponding to the short-term standard deviation, is estimated, and a long window $l_{l}$ in which the long-term standard deviation is calculated. The calculation of $\sigma_{l}$ is performed as median of the previously evaluated $\sigma_{s}$. The median operation was preferred to the average for its robustness to outliers that can be present as sudden high signal amplitude due to undetected non-respiratory motion. The duration of $l_{s}$ and $l_{l}$ was chosen considering the length of the targeted COBs, which varies from 3 to 10 seconds. Moreover, the short window should contain at least a single period of respiration to be able to detect also the RR. Since the minimum RR expected is $30 \mathrm{BPM}, l_{s}$ can be minimum 2 seconds. We arbitrarily decided to use a $l_{s}$ equal to 3 seconds. Furthermore, a too long $l_{l}$ will cause the threshold to not adapt dynamically to changes in the amplitude of the signal. While, a too short $l_{l}$ will result in adapting also during apneic events. Therefore, as a compromise $l_{l}$ was chosen to be equal to 11 seconds. More formally, let $\operatorname{resp}\left(n T_{s}\right)$ be the time domain signal after filtering obtained either from videos or from the reference, $n$ depends on the current window and it is defined as $n=0+(j-1), 1+(j-1), \ldots, N+(j-1)$. Where, $j$ indicates the current window, the number of samples per window is $N=l_{s} / T_{s}$ and $T_{s}$ the sampling time. Then the short-term standard deviation is evaluated according to:

$$
\sigma_{s}(j)=\sqrt{\frac{\sum_{n=0+(j-1)}^{N+(j-1)}\left(r e s p\left(n T_{s}\right)-\mu(j)\right)^{2}}{N}},
$$

where, $\mu(j)$ is the average of $\operatorname{resp}\left(n T_{s}\right)$. Thus, a value corresponding to the standard deviation of the time-domain signal will be obtained for each 3 seconds window. The long-term standard deviation will be evaluated on a window length $l_{l}$ equal to 11 seconds, however to reduce the delay in the detection, $\sigma_{l}$ is estimated with a fewer number of $\sigma_{s}$ until $j>H$ with $H=\frac{l_{l}}{T_{s}}$ :

$$
\sigma_{l}(j)= \begin{cases}\operatorname{median}_{1 \leq k \leq j-1}\left(\sigma_{s}(k)\right) & j \leq H \\ \underset{j-H \leq k \leq j-1}{\operatorname{median}}\left(\sigma_{s}(k)\right) & \text { otherwise. }\end{cases}
$$

In each window $\sigma_{s}(j)$ and $\sigma_{l}(j)$ are compared. If the ratio between the two standard deviations results in being lower than $33 \%$, the $j$-th window is considered to contain a $\mathrm{COB}$ and a binary template, $C D$, is created as follows:

$$
C D(j)= \begin{cases}1 & \sigma_{s}(j) \leq \sigma_{l}(j) / 3 \\ 0 & \text { otherwise }\end{cases}
$$

$C D(j)$ indicates if the window $j$ contains a COB. Figure 2 shows two examples, a signal containing $\mathrm{COBs}$ and one without.

Lee's method is applied on each video retrospectively on both CI and VRM signals. The method returns a probability of apnea, that is then converted to the weighted apnea duration as the area under the probability curve. The limit on the duration of the apneas detected, previously defined by Lee et al. as 5 seconds, is adjusted to this case making the smaller apnea detectable equal to 3 seconds, and obtaining therefore a second binary template for reference.

In the VRM respiration signal, since also motion information is available, cessations are not considered when motion unrelated to respiration is present and the standard deviation value $\sigma_{s}(j)$ for a j-th window containing motion is not considered in the calculation of the $\sigma_{l}(j)$. 

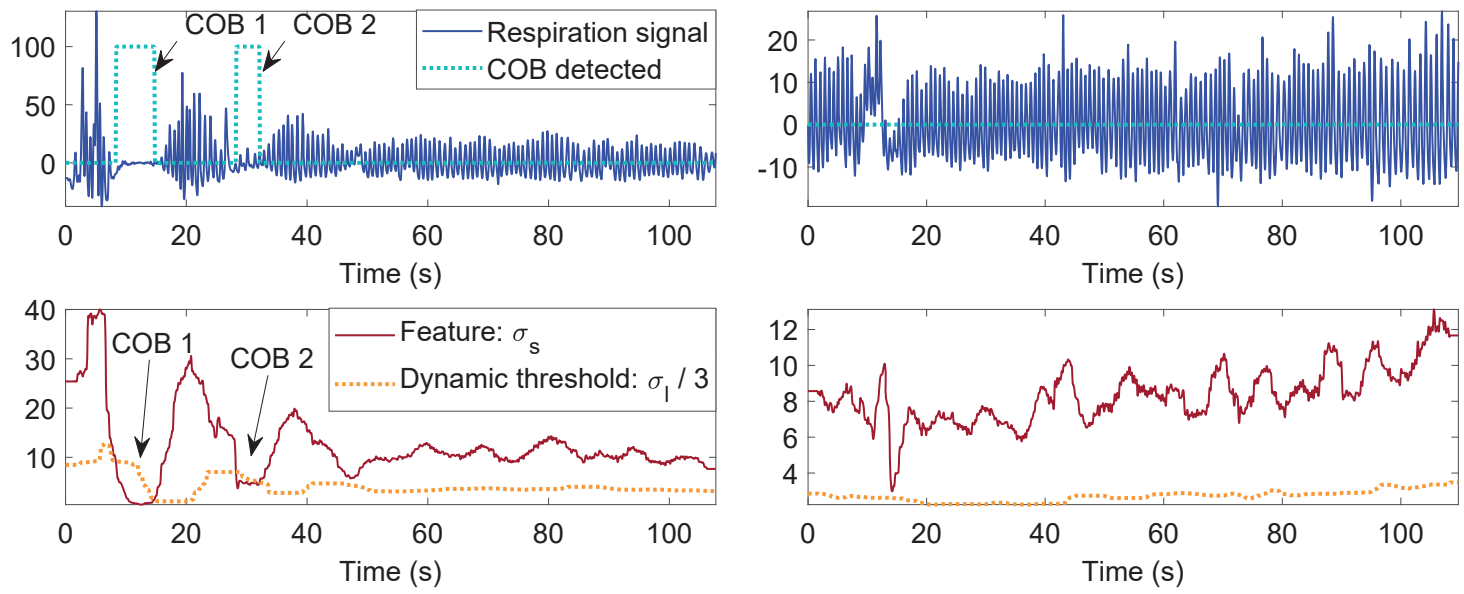

Figure 2. Examples of respiration signals, the two upper plots show a VRM respiration signal containing two cessations of breathing and a VRM respiration signal without cessations. The binary template, CD, labelled as "COB detected" has been multiplied for 100 for visualization purposes. The two plots in the bottom show the $\sigma_{s}$ used as feature for cessation of breathing detection and the threshold based on the $\sigma_{l}$.

\subsubsection{Respiration rate estimation}

In each 3 seconds window the respiration rate is estimated as the frequency corresponding to the peak in the spectrum for both VRM signal and CI. The spectrum is evaluated using FFT, zeropadding is performed reaching a number of samples equal to $120 \cdot 3 \cdot f_{s}$ with $f_{s}$ equal to the sampling frequency. Moreover, to compensate for small variations, the RRs obtained are filtered using a moving mean filter followed by a moving median filter each with a window size of half a second.

\subsubsection{Evaluation}

To compare respiration rates obtained with CI and VRM respiration signals the percentage of time in which the difference between the two is within \pm 6 BPM is used as metric. This percentage has been evaluated in each video and then averaged. Moreover, for fair comparison, the RRs estimated in windows containing COBs according to the annotations and those estimated in windows where motion of the infant unrelated to the respiration was detected from VRM algorithm have not been considered in the calculation of this metric.

To evaluate the difference between the COB detection algorithm proposed in this work and the one proposed by Lee et al. [24], sensitivity and specificity are calculated for each method using the manual annotation of the videos as reference. As defined in [9] sensitivity will be:

$$
S E=\frac{T T P}{T T P+T F N},
$$

with Time True Positive (TTP) and Time False Negative (TFN) being respectively the total duration of the time in- tervals with COBs detected correctly and with COBs incorrectly missed by the algorithm. And specificity will be:

$$
S P=\frac{T T N}{T T N+T F P},
$$

with Time True Negative (TTN) being the duration of the time intervals with no COBs in which there are no wrong detection while, Time False Positive (TFP) is the total length of the time segments with no COBs in which COBs are erroneously detected. Therefore, sensitivity represents the ability of the algorithm to correctly detect COBs when present whereas specificity is the ability to correctly exclude the presence of COBs particularly important to avoid false alarms. The accuracy can be therefore defined as:

$$
A C C=\frac{T T N+T T P}{T T N+T T P+T F P+T F N} .
$$

\subsection{Study design}

Videos were collected in the NICU of the Maxima Medical Center (MMC) in Veldhoven, The Netherlands. Two different setups were used for the data collection. Both studies received approval from MMC and one study also received approval from the Internal Committee for Biomedical Ethics in Philips Research (ICBE2013-41-3797). Informed parental consent was obtained for all the infants involved in the studies.

The videos were annotated by a single author, the COBs were annotated only when clearly visible in the video. In total, 5 infants were included, Table 1 shows the PostMenstrual Age (PMA) expressed as the gestational age plus the postnatal age, the total duration of the videos per infant, and the number of short apneic event annotated. The 


\begin{tabular}{c|cccc} 
ID & $\begin{array}{c}\text { PMA } \\
\text { (weeks) }\end{array}$ & $\begin{array}{c}\text { Number } \\
\text { of videos }\end{array}$ & $\begin{array}{c}\text { Total Duration } \\
(\text { min })\end{array}$ & $\begin{array}{c}\text { COB } \\
\text { annotated }\end{array}$ \\
\hline \hline 1 & $36+6.71$ & 10 & 16.5 & 11 \\
2 & $30+4.85$ & 20 & 34.3 & 4 \\
3 & $30+2.42$ & 10 & 49.8 & 31 \\
4 & $30+2.42$ & 10 & 46.2 & 24 \\
5 & $29+1.14$ & 9 & 43.6 & 21 \\
\hline \hline
\end{tabular}

Table 1. Video details and parameters of the infants in the dataset.

dataset includes both videos containing $\mathrm{COBs}$ and videos not containing any cessation events for control purposes. The videos have different duration going from 1 minute to 5 minutes reaching a total cumulative duration of $190.4 \mathrm{~min}-$ utes. In total 91 short apneic events were annotated, the average duration and standard deviation of the COBs population are $5.4 \pm 1.9$ seconds.

\subsection{Experimental setup}

The dataset compromises of videos collected with two different setups. In both cases the CI from the patient monitor (Philips MX800) was also acquired for reference purposes. In the first study a camera (UI-2220SE, IDS) was positioned using a tripod to have view of the infants chest/abdomen area, some videos were collected from the top and others from the side. The videos were collected under visible light conditions with a frame rate of 20 frames per second and with a resolution of $768 \times 576$ pixels. Since color information is not relevant for respiration-motion detection we used the raw gray-scale images. The videos were selected based on the quality of the reference signal and on the light conditions since the dataset also included measurements taken in particularly dark settings. Two infants (ID 1 and 2 in Table 1) were selected with a total video duration of 50.8 minutes.

The second setup included a monochrome visible light camera with the NIR filter removed (UI-22330SE, IDS) positioned on the incubator using suction cup mounting and visualizing an overview of the infant. NIR custom made illumination was used since the normal workflow of the NICU was not disrupted and the incubator was covered, as common practice, limiting the ambient light. The illumination unit comprised of LED arrays at three different wavelengths $(660,760$, and $810 \mathrm{~nm})$. The illumination level of all LEDs resulted in being around $0.2 \mathrm{~mW} / \mathrm{cm} 2$ at the skin level of a patient, definitely below the imposed limits (ANSI/AAMI/IEC 60601-2-21:2009). The videos were collected with a frame rate of 15 frames per second, a resolution of $608 \times 864$ pixels, and subjected to compression. In this case, the videos were selected only based on the quality

\begin{tabular}{cc|ccc} 
& & SE (\%) & SP (\%) & ACC (\%) \\
\hline \hline \multirow{2}{*}{ VRM } & Ours & 76.32 & $\mathbf{9 4 . 3 9}$ & $\mathbf{9 3 . 1 6}$ \\
& Lee’s method & $\mathbf{8 6 . 6 8}$ & 91.50 & 90.64 \\
\hline \multirow{2}{*}{ CI } & Ours & $\mathbf{8 3 . 1 5}$ & 96.97 & 96.00 \\
& Lee's method & 77.02 & $\mathbf{9 7 . 9 9}$ & $\mathbf{9 6 . 6 0}$
\end{tabular}

Table 2. Average sensitivity, specificity, and accuracy results obtained with the method proposed in this work, indicated as ours, and Lee et al. method.

of the reference signal. Using this setup, three infants are recorded as part of this dataset with a total video duration of 139.6 minutes.

\section{Results}

Table 2 contains the average results obtained for all the infants, sensitivity, specificity, and accuracy were estimated by comparing the detection of COBs performed with the method proposed in this work and Lee's method on both $\mathrm{CI}$ and VRM signals. The results were obtained using the parameters described in Section 2.1.2. In bold are indicated the best results comparing our method and Lee's. Figure 3 shows some examples of the obtained results. Figure 3 a contains an example where motion but no COB was present, while Figure $3 \mathrm{~b}$ shows the results obtained when three COBs were present. In the $\mathrm{CI}$ case, the $\mathrm{COBs}$ were all correctly detected by our methods, while the detection is incomplete in the video signal case for both the proposed method and the benchmark one.

\section{Discussion}

The method proposed in this work was proven to be able to detect short apneic events. Preliminary results were obtained with videos of five infants containing 91 COBs in total. The comparison between our method and the one previously proposed by Lee et al. shows that our method resulted in higher specificity and accuracy for the respiration signals obtained on the videos, while the opposite happens in the chest impedance case. Moreover, the results were always higher in the case of Chest Impedance signal compared to the VRM signal using our COB detector. This is most likely due to the noisiness of the signals, the VRM respiration signal relies on the correct detection of the ROI, that can be momentarily lost after strong movements causing low amplitude in the signal that can be misinterpreted as COBs. On average for both CI and VRM signals our method reached an accuracy of $94.6 \%$ against the $93.5 \%$ of Lee's method. Considering the high false alarm rate already present in the NICUs [20] it is of paramount importance to prefer specificity to sensitivity especially in the short apnea cases for which 


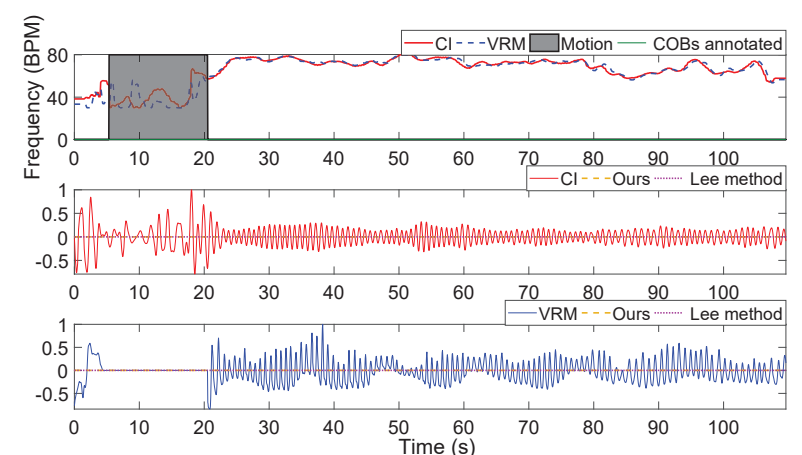

(a)

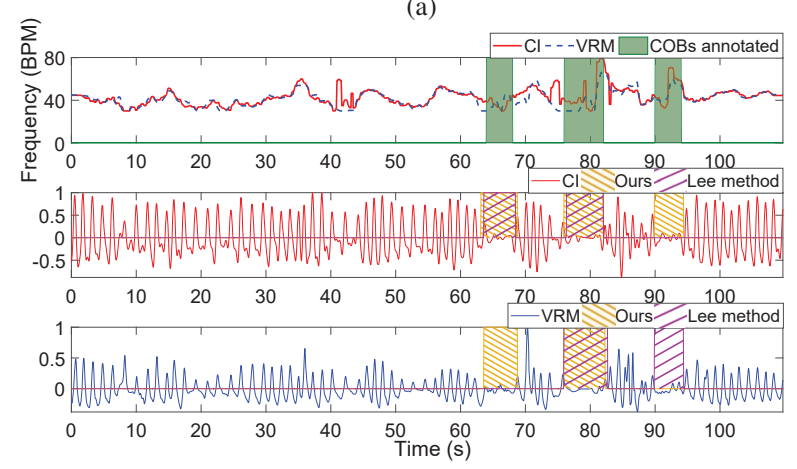

(b)

Figure 3. Example of results obtained: (a) CI and VRM respiration signal and RR when motion unrelated to respiration is detected; (b) case with $\mathrm{COB}$ annotated. The amplitudes of the respiration time-domain signals were scaled to 1 for visualization.

clinical relevance is still under discussion. Moreover, our method is able to work in an on-line fashion while, Lee et al. claim that their method requires several minutes of clean signal to work accurately and that an adaptation is needed for on-line detection [24], this is mostly due to the filtering and removal of baseline from the signal before the estimation of the moving standard deviation, on which the parameters of the Fermi function were optimized. Our method is also based on standard deviation but proved to work with a sliding window of 3 seconds, making COB rapidly detectable by the system.

Moreover, the respiration rate obtained with the video signal and the CI as reference were compared. The RR extracted from the VRM signal is $75 \%$ of the time within 6 BPM from the Chest Impedance one. Higher errors were obtained for infants with ID 3,4, and 5, this can be due to the compression of the videos and/or to a higher number of events with small motions unrelated to respiration. It should be considered that most of the studies using frequency-based RR detection used windows ranging from 8 to 20 seconds $[32,3,17]$ in our case the 3 second window in which the FFT was performed leads to fast estimations but can also cause higher errors due to the poor frequency resolution.
This work introduced a new method for the detection of short apneas that can work on-line. The results are still considered preliminary, first of all the cessations of breathing were not annotated by an expert, however the use of videos and not of CI for the annotations makes the result less subjective. Moreover, also longer apnea should be considered, there is no suggestion that such a method would not work for a different $\mathrm{COB}$ population, however, parameters such as $l_{l}$ would need to be adjusted. The parameters used in this work were chosen arbitrarily or based on reasoning and the same parameters were chosen for CI and VRM signals, an optimization of these parameters could lead to improved results.

VRM delivered a respiration signal from videos with varying orientations and settings, e.g. containing motion or with infants covered. The algorithm is characterized by a set of empirically chosen parameters, we believe that adjusting the parameters could lead to improved respiration signal thanks to a more accurate detection of unwanted motion and a faster adaptation of the ROI following big movements.

\section{Conclusion}

The method described in this study proved in being able to detect short apneic events yielding an accuracy equal to $93.16 \%$ in the video signal case. The method can on-line detect cessations of breathing with a low latency and it is based on the comparison of short-term and long-term standard deviations. The detection of such short apneic events could lead to apnea prediction preventing hypoxic damages in infants.

\section{Funding}

This research was performed within the Alarm-Limiting AlgoRithm-based Monitoring (ALARM) project funded by Nederlandse Organisatie voor Wetenschappelijk Onderzoek (NWO) grant number 15345.

\section{Acknowledgments}

The authors are grateful for the help and contributions of Dr. Deedee Kommers, Dr. Mark van Gastel, Eng. Rik Janssen, and Eng. Shakith Fernando.

\section{References}

[1] L. A. Aarts, V. Jeanne, J. P. Cleary, C. Lieber, J. S. Nelson, S. B. Oetomo, and W. Verkruysse. Non-contact heart rate monitoring utilizing camera photoplethysmography in the neonatal intensive care unit-a pilot study. Early human development, 89(12):943-948, 2013. 1

[2] A. K. Abbas, K. Heimann, K. Jergus, T. Orlikowsky, and S. Leonhardt. Neonatal non-contact respiratory monitoring 
based on real-time infrared thermography. Biomedical engineering online, 10(93):1-17, 2011. 1

[3] D. Alinovi, G. Ferrari, F. Pisani, and R. Raheli. Respiratory rate monitoring by video processing using local motion magnification. In 2018 26th European Signal Processing Conference (EUSIPCO), pages 1780-1784. IEEE, 2018. 2, 6

[4] A. Bekele, S. Nizami, Y. S. Dosso, C. Aubertin, K. Greenwood, J. Harrold, and J. R. Green. Real-time neonatal respiratory rate estimation using a pressure-sensitive mat. In 2018 IEEE International Symposium on Medical Measurements and Applications (MeMeA), pages 1-5. IEEE, 2018. 1

[5] E. A. Bernal, L. K. Mestha, and E. Shilla. Non contact monitoring of respiratory function via depth sensing. In IEEEEMBS International Conference on Biomedical and Health Informatics (BHI), pages 101-104. IEEE, 2014. 1

[6] M. Bester, D. Van Den Heever, R. Joshi, and K. Dellimore. A study of short cessations and temporal dynamics of breathing in preterm infants. In 2018 3rd Biennial South African Biomedical Engineering Conference (SAIBMEC), pages 14. IEEE, 2018. 1, 2

[7] R. T. Brouillette, A. S. Morrow, D. E. Weese-Mayer, and C. E. Hunt. Comparison of respiratory inductive plethysmography and thoracic impedance for apnea monitoring. The Journal of pediatrics, 111(3):377-383, 1987. 1

[8] V. Cardot, K. Chardon, P. Tourneux, S. Micallef, E. Stephan, A. Leke, V. Bach, J.-P. Libert, and F. Telliez. Ventilatory response to a hyperoxic test is related to the frequency of short apneic episodes in late preterm neonates. Pediatric research, 62(5):591-596, 2007. 1

[9] L. Cattani, D. Alinovi, G. Ferrari, R. Raheli, E. Pavlidis, C. Spagnoli, and F. Pisani. A wire-free, non-invasive, lowcost video processing-based approach to neonatal apnoea detection. In 2014 IEEE Workshop on Biometric Measurements and Systems for Security and Medical Applications (BIOMS) Proceedings, pages 67-73. IEEE, 2014. 2, 4

[10] M. T. Clark, B. D. Vergales, A. O. Paget-Brown, T. J. Smoot, D. E. Lake, J. L. Hudson, J. B. Delos, J. Kattwinkel, and J. R. Moorman. Predictive monitoring for respiratory decompensation leading to urgent unplanned intubation in the neonatal intensive care unit. Pediatric research, 73(1):104-110, 2013. 2

[11] J. M. Di Fiore. Neonatal cardiorespiratory monitoring techniques. In Seminars in neonatology, volume 9, pages 195203. Elsevier, 2004. 1

[12] E. C. Eichenwald et al. Apnea of prematurity. Pediatrics, 137(1):1-7, 2016. 1

[13] D. E. Elder, A. J. Campbell, and D. Galletly. Current definitions for neonatal apnoea: are they evidence based? Journal of paediatrics and child health, 49(9):388-396, 2013. 1

[14] K. Fairchild, M. Mohr, A. Paget-Brown, C. Tabacaru, D. Lake, J. Delos, J. R. Moorman, and J. Kattwinkel. Clinical associations of immature breathing in preterm infants: part 1central apnea. Pediatric research, 80(1):21-27, 2016. 1

[15] X. Huang, L. Sun, T. Tian, Z. Huang, and E. Clancy. Realtime non-contact infant respiratory monitoring using uwb radar. In 2015 IEEE 16th International Conference on Communication Technology (ICCT), pages 493-496. IEEE, 2015. 1

[16] R. Janssen, W. Wang, A. Moço, and G. de Haan. Video-based respiration monitoring with automatic region of interest detection. Physiological measurement, 37(1):100-114, 2015. 2, 3

[17] J. Jorge, M. Villarroel, S. Chaichulee, A. Guazzi, S. Davis, G. Green, K. McCormick, and L. Tarassenko. Non-contact monitoring of respiration in the neonatal intensive care unit. In 2017 12th IEEE International Conference on Automatic Face \& Gesture Recognition (FG 2017), pages 286-293. IEEE, 2017. 2, 6

[18] J. Jorge, M. Villarroel, S. Chaichulee, K. McCormick, and L. Tarassenko. Data fusion for improved camera-based detection of respiration in neonates. In Optical Diagnostics and Sensing XVIII: Toward Point-of-Care Diagnostics, 1050112. International Society for Optics and Photonics, 2018. 2

[19] R. Joshi, B. Bierling, L. Feijs, C. van Pul, and P. Andriessen. Monitoring the respiratory rate of preterm infants using an ultrathin film sensor embedded in the bedding: a comparative feasibility study. Physiological measurement, 40(4):1-8, 2019. 1

[20] R. Joshi, H. van de Mortel, L. Feijs, P. Andriessen, and C. van Pul. The heuristics of nurse responsiveness to critical patient monitor and ventilator alarms in a private room neonatal intensive care unit. PloS one, 12(10):1-14, 2017. 5

[21] J. D. Kim, W. H. Lee, Y. Lee, H. J. Lee, T. Cha, S. H. Kim, K.-M. Song, Y.-H. Lim, S. H. Cho, S. H. Cho, et al. Non-contact respiration monitoring using impulse radio ultrawideband radar in neonates. Royal Society Open Science, 6(6):1-11, 2019. 1

[22] J. H. Klaessens, M. van den Born, A. van der Veen, J. Sikkens-van de Kraats, F. A. van den Dungen, and R. M. Verdaasdonk. Development of a baby friendly non-contact method for measuring vital signs: first results of clinical measurements in an open incubator at a neonatal intensive care unit. In Advanced Biomedical and Clinical Diagnostic Systems XII, 89351P. International Society for Optics and Photonics, 2014. 1

[23] N. Koolen, O. Decroupet, A. Dereymaeker, K. Jansen, J. Vervisch, V. Matic, B. Vanrumste, G. Naulaers, S. Van Huffel, and M. De Vos. Automated respiration detection from neonatal video data. In $\operatorname{ICPRAM}(2)$, pages 164-169, 2015. 1

[24] H. Lee, C. G. Rusin, D. E. Lake, M. T. Clark, L. Guin, T. J. Smoot, A. O. Paget-Brown, B. D. Vergales, J. Kattwinkel, J. R. Moorman, et al. A new algorithm for detecting central apnea in neonates. Physiological measurement, 33(1):1-17, 2011. 1, 2, 3, 4, 6

[25] M. A. Mohr, K. D. Fairchild, M. Patel, R. A. Sinkin, M. T. Clark, J. R. Moorman, D. E. Lake, J. Kattwinkel, and J. B. Delos. Quantification of periodic breathing in premature infants. Physiological measurement, 36(7):1415-1427, 2015. 2

[26] M. A. Mohr, B. D. Vergales, H. Lee, M. T. Clark, D. E. Lake, A. C. Mennen, J. Kattwinkel, R. A. Sinkin, J. R. Moorman, 
K. D. Fairchild, et al. Very long apnea events in preterm infants. Journal of Applied Physiology, 118(5):558-568, 2014. 2

[27] C. B. Pereira, K. Heimann, B. Venema, V. Blazek, M. Czaplik, and S. Leonhardt. Estimation of respiratory rate from thermal videos of preterm infants. In 2017 39th Annual International Conference of the IEEE Engineering in Medicine and Biology Society (EMBC), pages 3818-3821. IEEE, 2017. 1

[28] S. A. Pullano, I. Mahbub, M. G. Bianco, S. Shamsir, S. K. Islam, M. S. Gaylord, V. Lorch, and A. S. Fiorillo. Medical devices for pediatric apnea monitoring and therapy: past and new trends. IEEE reviews in biomedical engineering, 10:199-212, 2017. 1

[29] H. Rehouma, R. Noumeir, W. Bouachir, P. Jouvet, and S. Essouri. 3d imaging system for respiratory monitoring in pediatric intensive care environment. Computerized Medical Imaging and Graphics, 70:17-28, 2018. 1

[30] S. Reuter, C. Moser, and M. Baack. Respiratory distress in the newborn. Pediatrics in review, 35(10):417-429, 2014. 3

[31] S. J. Rhoads, A. L. Green, S. D. Lewis, and L. Rakes. Challenges of implementation of a web-camera system in the neonatal intensive care unit. Neonatal Network, 31(4):223228, 2012. 1

[32] M. van Gastel, S. Stuijk, and G. de Haan. Robust respiration detection from remote photoplethysmography. Biomedical optics express, 7(12):4941-4957, 2016. 2, 6

[33] M.-F. Vecchierini, L. Curzi-Dascalova, H. Trang-Pham, J. Bloch, and C. Gaultier. Patterns of eeg frequency, movement, heart rate, and oxygenation after isolated short apneas in infants. Pediatric research, 49(2):220-226, 2001. 1

[34] B. D. Vergales, A. O. Paget-Brown, H. Lee, L. E. Guin, T. J. Smoot, C. G. Rusin, M. T. Clark, J. B. Delos, K. D. Fairchild, D. E. Lake, et al. Accurate automated apnea analysis in preterm infants. American journal of perinatology, 31(02):157-162, 2014. 2

[35] M. Villarroel, A. Guazzi, J. Jorge, S. Davis, P. Watkinson, G. Green, A. Shenvi, K. McCormick, and L. Tarassenko. Continuous non-contact vital sign monitoring in neonatal intensive care unit. Healthcare technology letters, 1(3):87-91, 2014. 1 